\title{
Формирование InAs/GaP-гетероструктур с квантовыми ямами на подложках кремния методом молекулярно-лучевой эпитаксии
}

\author{
(ㄷ Д.С. Абрамкин ${ }^{1,2}$, М.О. Петрушков ${ }^{1}$, Е.А. Емельянов ${ }^{1}$, А.В. Ненашев ${ }^{1,2}$, M.Ю. Есин $^{1}$, А.В. Васев ${ }^{1}$, \\ М.А. Путято ${ }^{1}$, Д.Б. Богомолов ${ }^{1}$, А.К. Гутаковский ${ }^{1,2}$, В.В. Преображенский ${ }^{1}$ \\ ${ }^{1}$ Институт фризики полупроводников им. А.В. Ржанова Сибирского отделения Российской академии наук, \\ 630090 Новосибирск, Россия \\ ${ }^{2}$ Новосибирский государственный университет, \\ 630090 Новосибирск, Россия \\ E-mail: dalamber.07@mail.ru
}

Поступила в Редакцию 7 октября 2020 г.

В окончательной редакции 13 октября 2020 г.

Принята к публикации 13 октября 2020 г.

\begin{abstract}
Продемонстрирована возможность формирования псевдоморфно-напряженной квантовой ямы, состоящей из четверного твердого раствора $\mathrm{In}_{x} \mathrm{Ga}_{1-x} \mathrm{As}_{y} \mathrm{P}_{1-y}$, при осаждении InAs на поверхность $\mathrm{GaP} / \mathrm{Si}$ эпитаксиального слоя с развитым рельефом поверхности. Проведены исследования квантовой ямы методами просвечивающей электронной микроскопии и спектроскопии стационарной фотолюминесценции. Обнаружено формирование двух различных сегментов квантовой ямы, отличающихся толщиной и составом твердого раствора $\operatorname{In}_{x} \mathrm{Ga}_{1-x} \mathrm{As}_{y} \mathrm{P}_{1-y}$, при этом увеличение толщины квантовой ямы сопровождается снижением содержания атомов In и As. Латеральные размеры сегментов квантовой ямы составляют не менее 20 нм. Сегментам квантовой ямы соответствуют две различные полосы низкотемпературной фотолюминесценции. Наблюдаемые явления объяснены в рамках предположения о перестройке поверхности под действием упругих деформаций при гетероэпитаксии InAs на террасированной поверхности GaP.
\end{abstract}

Ключевые слова: молекулярно-лучевая эпитаксия, InAs/GaP-квантовые ямы, $\mathrm{A}^{\mathrm{III}} \mathrm{B}^{\mathrm{V}}$ на кремнии, морфология поверхности, фотолюминесценция, перемешивание материалов, упругие деформации.

DOI: 10.21883/FTP.2021.02.50500.9529

\section{1. Введение}

Монолитная интеграция оптоэлектронных устройств, создаваемых на базе материалов $\mathrm{A}^{\mathrm{III}} \mathrm{B}^{\mathrm{V}}$, в кремниевые микросхемы позволит совершить качественный технологический скачок в области передачи и обработки информации [1-4]. Одним из самых перспективных материалов с точки зрения интеграции $\mathrm{A}^{\mathrm{III}} \mathrm{B}^{\mathrm{V}}$ структур в кремниевые устройства является $\mathrm{GaP}$, практически согласованный с $\mathrm{Si}$ по значению постоянной решетки. К сожалению, $\mathrm{GaP}$ является непрямозонным материалом [5], что ограничивает его применение в качестве активной области светоизлучающих структур из-за низкой вероятности излучательных переходов. Однако формирование в $\mathrm{GaP}$ низкоразмерных гетероструктур с квантовыми ямами (КЯ) и квантовыми точками (КТ), состоящих из узкозонных материалов, позволяет сместить основное электронное состояние в них к центру зоны Бриллюэна и увеличить эффективность излучательной рекомбинации носителей заряда. Так, например, в работах [6,7] показана возможность формирования прямозонных InGaAs/GaP KT

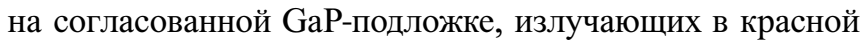
области спектра (1.7-1.8 эВ) при комнатной температуре. Кроме того, продемонстрирована эффективная люминесценция ненапряженных $\mathrm{GaAs} / \mathrm{GaP}$ KT при комнатной температуре, также выращенных на согласованной $\mathrm{GaP}$-подложке и являющихся прямозонными объектами [8]. Несмотря на сложившееся достаточно полное по- нимание особенностей формирования, строения и электронного спектра $\mathrm{GaAs} / \mathrm{GaP}[8,9]$ и $\mathrm{InGaAs} / \mathrm{GaP}[10,11]$, сформированных на согласованных подложках $\mathrm{GaP}$, имеется ограниченное число работ, посвященных формированию таких структур на Si-подложках. Так, в работе [12] сообщается о получении $\mathrm{InGaAs} / \mathrm{GaP} / \mathrm{Si}$-гетероструктур с КТ, излучающих при комнатной температуре, а в работе [13] GaAs/GaP/Si-гетероструктур с КЯ, сравнимых по эффективности низкотемпературной люминесценции с аналогичными структурами, выращенными на согласованной GaP-подложке. Особенности процессов формирования и кристаллического строения таких гетероструктур, обусловленные ростом на искусственных $\mathrm{GaP} / \mathrm{Si}$ подложках, в литературе практически не обсуждаются. В данной работе рассматривается формирование и строение InAs/GaP-гетероструктур, выращенных методом молекулярно-лучевой эпитаксии (МЛЭ) на кремниевых подложках.

\section{2. Рост гетероструктур}

Гетероструктуры InAs/GaP/Si выращивались методом МЛЭ на модернизированной установке „Штат“, оборудованной вентильными источниками молекул $\mathrm{As}_{2}$ и $\mathrm{P}_{2} \mathrm{c}$ зонами крекинга и тигельными источниками $\mathrm{Ga}$, In и $\mathrm{Si}$. Структурное состояние поверхности подложки и эпитаксиальных слоев контролировались в процессе роста методом дифракции быстрых электронов на отражение. 
Плотности потоков веществ измерялись ионизационным вакуумметром, датчик которого помещался на позицию подложки. Рост буферных слоев $\mathrm{GaP} / \mathrm{Si}$ толщиной 500 нм проходил на $\mathrm{Si}$-подложках ориентации (001), отклоненных на $6^{\circ}$ в направлении [110]. Отклоненные подложки использовались в целях подавления образования антифазных дефектов $[14,15]$. Окисный слой с поверхности $\mathrm{Si}$ удалялся при температуре подложки $\left(T_{S}\right) 720^{\circ} \mathrm{C}$ в потоке атомов кремния в течение 10 мин. Затем подложка отжигалась 30 мин в условиях сверхвысокого вакуума при температуре $800^{\circ} \mathrm{C}$ для объединения моноатомных ступеней в двухатомные. Начальные стадии роста $\mathrm{GaP} / \mathrm{Si}$ (первые 6 монослоев вещества) проводились в режиме атомно-слоевой эпитаксии при $T_{s}=300^{\circ} \mathrm{C}$. При росте следующего слоя в слабом потоке фосфора (отношение $\mathrm{V} / \mathrm{III}<1$ ) осаждалось 4 монослоя $(\mathrm{MC})$ атомов $\mathrm{Ga}$, затем поверхность выдерживалась в потоке фосфора в течение 20 с. Эта процедура повторялась, пока общая толщина пленки не составила 55 нм. Затем $T_{s}$ повышалась до $500^{\circ} \mathrm{C}$, и в том же режиме выращивался слой $\mathrm{GaP}$ толщиной 150 нм. Последующий слой, толщиной 300 нм, был выращен со скоростью $1 \mathrm{MC} / \mathrm{c}$ при $T_{s}=600^{\circ} \mathrm{C}$ в режиме двумерно-слоевого роста при одновременном осаждении материалов III и V групп с соотношением потоков V/III > 2. Перед формированием InAs/GaP-гетероструктур рост останавливался, и в потоке фосфора подложка охлаждалась до $520^{\circ} \mathrm{C}$. Гетероструктуры выращивались путем осаждения InAs в количестве, эквивалентном 2 монослоям (MC) вещества, со скоростью $0.2 \mathrm{MC} / \mathrm{c}$. Осаждение InAs проходило на поверхность $\mathrm{GaP}$ с поверхностной реконструкцией $(2 \times 4)$. Сразу же после осаждения InAs-структур наращивался 50-нм слой $\mathrm{GaP}$ при той же $T_{s}$.

Морфология поверхности $\mathrm{GaP} / \mathrm{Si}$, на которой проходил рост InAs-структур, исследована методом атомносиловой микроскопии (ACM) на микроскопе Solver P47 (NT-MDT). Кристаллическое строение гетероструктур исследовано методом просвечивающей электронной микроскопии (ПЭМ) с помощью микроскопа Titan 80-300 (FEI Company). Стационарная фотолюминесценция (ФЛ) возбуждалась излучением $\mathrm{GaN}$-лазерного диода с энергией кванта 3.06 эВ и плотностью мощности $\left(P_{\mathrm{ex}}\right)$, варьируемой в диапазоне $0.6 \mathrm{MBт} / \mathrm{cm}^{2}-25 \mathrm{Bт} / \mathrm{cm}^{2}$. Излучение ФЛ собиралось с участка структуры с характерным латеральным размером $\sim 300$ мкм и анализировалось с помощью спектрографа Acton Advanced SP2500A, оборудованного ПЗС-камерой с азотным охлаждением. Измерения проводились при температуре $5 \mathrm{~K}$.

\section{3. Результаты}

\section{1. Морфология поверхности GaP}

Проведены исследования морфологии поверхности слоев $\mathrm{GaP} / \mathrm{Si}$, выращенных в аналогичных условиях, что и буферные слои, на которых формировались гетероструктуры InAs/GaP/Si. Изображение поверхности, полученное методом АСМ, представлено на рис. 1.

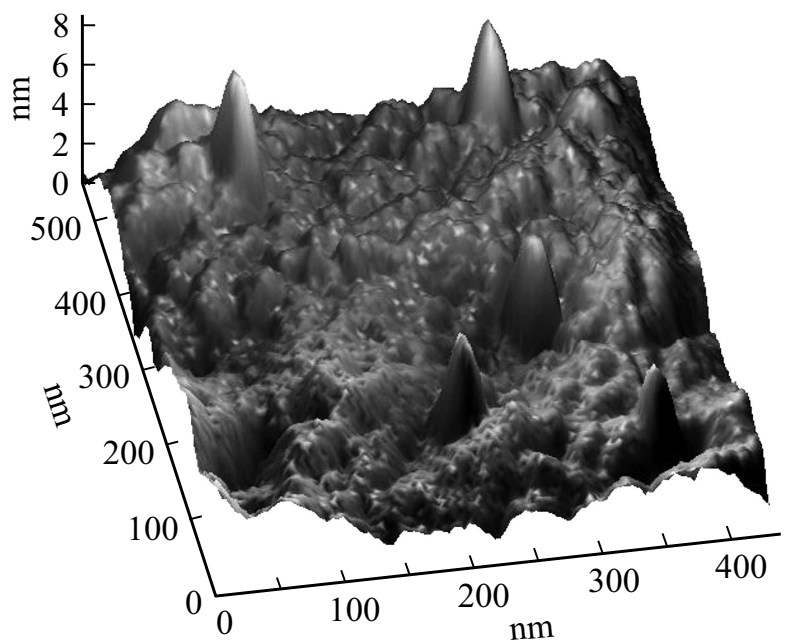

Рис. 1. АСМ-изображение поверхности $\mathrm{GaP} / \mathrm{Si}-$ буферного слоя.

Как видно из рис. 1, поверхность характеризуется развитой морфологией. Статистический анализ АСМизображения размером $10 \times 10$ мкм показывает, что можно выделить следующие особенности морфологии: (1) плавные „волны“ рельефа поверхности, с характерным латеральным размером 150-250 нм и вертикальным перепадом $\sim 10$ нм; (2) крупные „островки“, латеральные размеры которых лежат в пределах 30-70 нм, а вертикальные составляют $\sim 7$ нм; (3) мелкие „островки“ с латеральными размерами 15-40 нм и высотой не более $4 \mathrm{Hм}$.

\section{2. Просвечивающая электронная микроскопия}

Поперечные срезы InAs/GaP гетероструктур были изучены методом ПЭМ. Как видно из ПЭМ-изображения, представленного на рис. 2, $a$, в слоях $\mathrm{GaP}$ присутствуют прорастающие дислокации плотностью $10^{8}-10^{9} \mathrm{~cm}^{-2}$. Предположительно, введение дислокаций в слой $\mathrm{GaP}$, согласованный по постоянным решетки (разница $\sim 0.3 \%$ ) с Si-подложкой в процессе высокотемпературной гетерэпитаксии, может происходить при остывании гетероструктуры $\mathrm{GaP} / \mathrm{Si}$ за счет появления механических напряжений из-за разности коэффициентов температурного расширения $\mathrm{GaP}$ и $\mathrm{Si}[5,16]$ и (или) в ходе срастания трехмерных островков на начальных стадиях роста пленки $\mathrm{GaP} / \mathrm{Si}$ [17]. Из рис. $2, a$ видно, что в InAs/GaP-структурах происходит формирование КЯ и не наблюдается образования самоорганизованных КТ. В работе [18] сообщается, что осаждение уже $1 \mathrm{MC}$ InAs на поверхность GaP приводит к самоорганизации КТ по механизму Фальмера-Вебера. Как мы считаем, наблюдаемое в нашем случае подавление формирования КТ связано со снижением латерального массопереноса, которое может быть вызвано как отсутствием ростовой паузы [7], так и осложнением поверхностной диффузии 

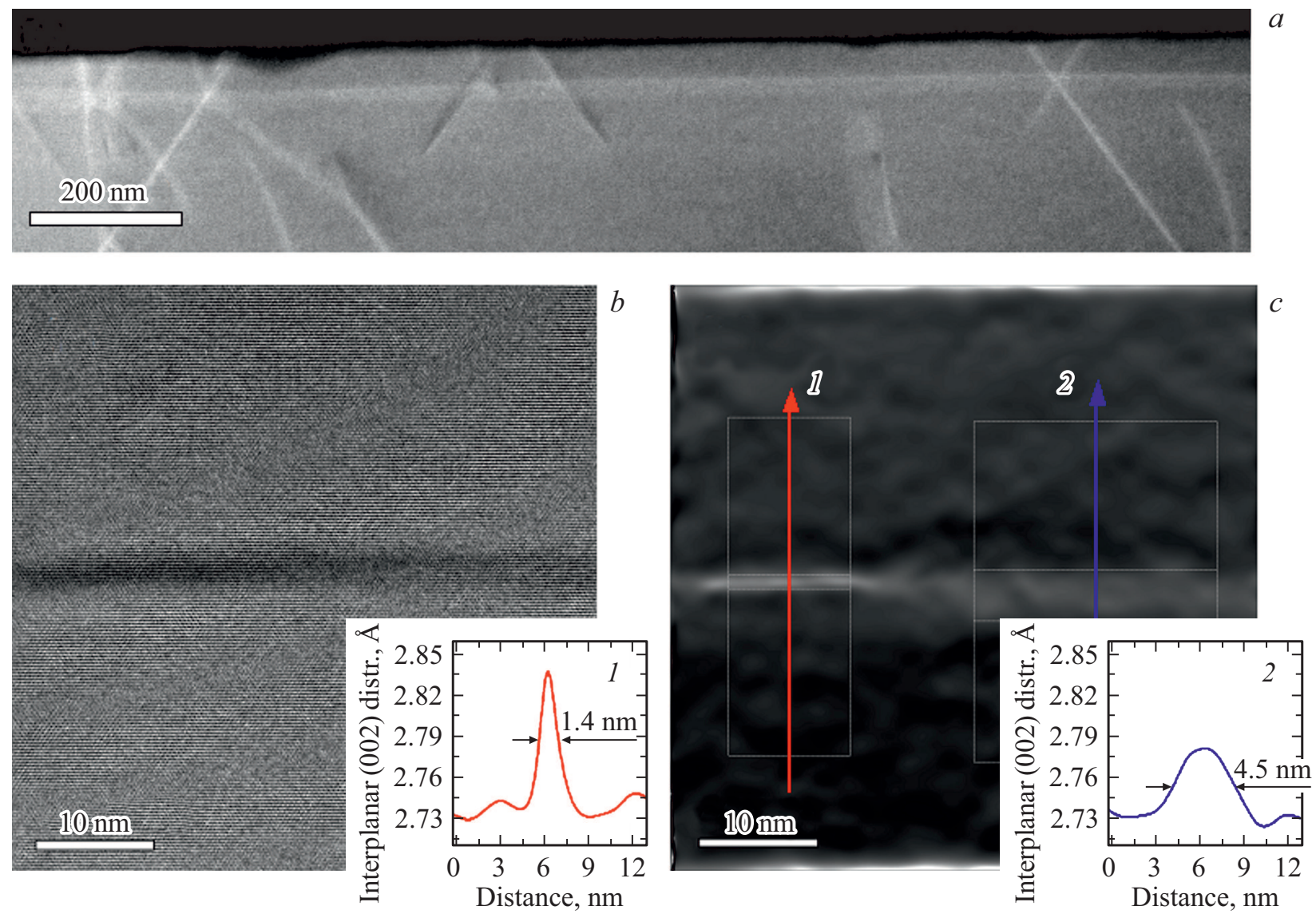

Рис. 2. $a-$ ПЭМ-изображение поперечного среза InAs/GaP-гетеростуктуры, полученное с низким разрешением. $b-$ изображение поперечного среза InAs/GaP-гетероструктуры, полученное методом высокоразрешающей просвечивающей электронной микроскопии (ВРЭМ). $c$ - карта пространственного распределения межплоскостного расстояния для плоскостей (002), построенная для того же участка КЯ, который представлен на рис. 2, $b$. На вставке к рис. $2, b$ представлена зависимость межплоскостного расстояния для плоскостей (002) от координаты вдоль оси роста (направление обозначено стрелкой 1 на рис. $2, c)$. На вставке к рис. 2, $c$ представлена зависимость межплоскостного расстояния для плоскостей (002) от координаты вдоль оси роста (направление обозначено стрелкой 2 на рис. 2,c). Тонкие линии на рис. 2,c показывают области изображений, по которым проведено усреднение значений межплоскостного расстояния.

адатомов по поверхности $\mathrm{GaP}$ с развитым рельефом. Далее мы будем обсуждать строение КЯ на участках, далеких от мест пересечения КЯ и дислокаций, прорастающих из буферных $\mathrm{GaP} / \mathrm{Si}-$ слоев.

Как видно, из изображений, полученных методом высокоразрешающей просвечивающей электронной микроскопии (ВРЭМ) (см. рис. 2, $b$ ), КЯ не содержит протяженных дефектов, которые могли бы обеспечить релаксацию упругих деформаций, а значит, можно утверждать, что КЯ псевдоморфно напряжена. Анализ изображений методом геометрической фазы позволил построить карту пространственного распределения межплоскостного расстояния для плоскостей типа (002) $d_{002}$, представленную на рис. 2,c. На картах видно, что наблюдаются участки с существенно отличающейся толщиной InAs/GaP КЯ. Латеральные размеры участков КЯ составляют не менее 20 нм. Были построены зависимости межплоскостного расстояния $d_{002}$ от координаты вдоль оси роста для участков InAs/GaP КЯ различной толщины. Зависимость для участков КЯ, обозначенных на рис. 2, с как 1 и 2, представлена на вставках к рис. 2, $c$ и $b$ соответственно. Видно, что участки КЯ характеризуются толщиной 1.4 и 4.5 нм, в то время как величина межплоскостного расстояния $d_{002}$ достигает значений 2.84 и $2.78 \AA$ соответственно.

Анализ величины межплоскостного расстояния материала КЯ позволил нам оценить состав твердого раствора, из которого состоят КЯ. Для псевдоморфно-напряженного тонкого слоя, согласованного с материалом матрицы в плоскости структуры, величина деформации в плоскости структуры равна (см. работу [19]):

$$
\varepsilon_{x x}=\frac{a_{\mathrm{GaP}}}{a_{0}}-1,
$$

где $a_{\mathrm{GaP}}-$ постоянная решетки $\mathrm{GaP}, a_{0}-$ постоянная решетки ненапряженного материала КЯ. В рамках приближения сплошной среды [19] деформации в направлении оси роста $\varepsilon_{z z}$ и в плоскости структуры $\varepsilon_{x x}$ связаны 


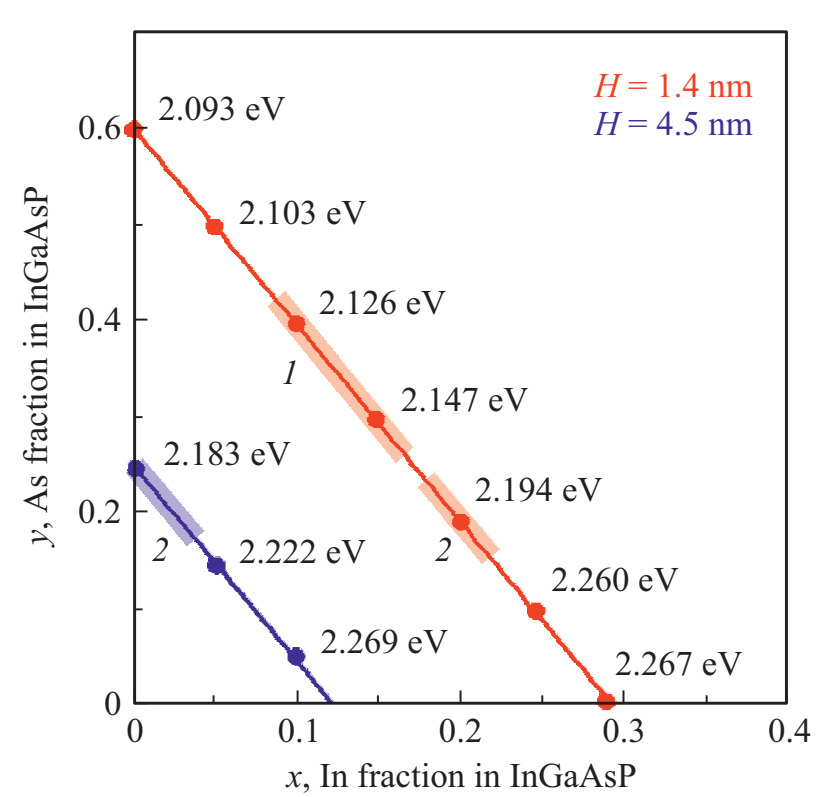

Рис. 3. Области возможных значений твердого раствора $\mathrm{In}_{x} \mathrm{Ga}_{1-x} \mathrm{As}_{y} \mathrm{P}_{1-y} / \mathrm{GaP}$ КЯ, рассчитанные на основе данных ПЭМ. Красная и синяя линии соответствуют областям значений твердого раствора для участков КЯ толщиной 1.4 и 4.5 нм соответственно. Точки указывают на значения состава твердого раствора, для которых были проведены расчеты энергии оптического перехода КЯ. Расчетные значения энергии оптического перехода подписаны на рисунке. Закрашенные области, подписанные 1 и 2 , соответствуют областям значений состава твердого раствора, определенным из сопоставления расчетных энергий оптического перехода с данными ФЛ для полос 1 и 2 соответственно.

следующим соотношением [19]:

$$
\varepsilon_{z z}=-2 \frac{C_{12}}{C_{11}} \varepsilon_{x x}
$$

где $C_{12}$ и $C_{11}-$ упругие константы материала КЯ. В свою очередь деформация $\varepsilon_{z z}$ равна [19]:

$$
\varepsilon_{z z}=\frac{a_{\mathrm{TEM}}}{a_{0}}-1,
$$

где $a_{\text {TЕм }}-$ постоянная решетки напряженной КЯ в направлении вдоль оси роста, определяемая из эксперимента (равная удвоенному межплоскостному расстоянию $\left.d_{002}\right)$. Таким образом, можно определить постоянную решетки ненапряженного материала КЯ:

$$
a_{0}=\frac{a_{\mathrm{TEM}}+2 a_{\mathrm{GaP}} C_{12} / C_{11}}{1+2 C_{12} / C_{11}} .
$$

В соответствии с законом Вегарда для постоянной решетки твердого раствора для $a_{0} \mathrm{In}_{x} \mathrm{Ga}_{1-x} \mathrm{As}_{y} \mathrm{P}_{1-y}$ КЯ равна [5]:

$$
\begin{aligned}
a_{0}= & x y a_{\mathrm{InAs}}+(1-x) y a_{\mathrm{GaAs}}+x(1-y) a_{\mathrm{InP}} \\
& +(1-x)(1-y) a_{\mathrm{GaP}} .
\end{aligned}
$$

Это соотношение позволяет определить область составов $(x, y)$ твердого раствора, из которого состоят участки КЯ, видимые на ПЭМ-изображениях. Отметим, что разброс значений соотношения $2 C_{12} / C_{11}$ для материалов InAs, InP, GaAs и GaP (0.883-1.109 [5]) не оказывает заметного влияния на результат расчета, и поэтому было использовано значение, среднее $2 C_{12} / C_{11}=1.0015$.

Результаты расчетов возможных значений состава твердого раствора КЯ приведены на рис. 3. Области возможных значений составов твердых растворов $\mathrm{In}_{x} \mathrm{Ga}_{1-x} \mathrm{As}_{y} \mathrm{P}_{1-y}$ для участков КЯ толщиной 1.4 и 4.5 нм выделены красной и синей линиями соответственно. Область значений состава твердого раствора для „узкой“ части $\mathrm{InGaAsP} / \mathrm{GaP}$ КЯ находится в пределах значений $x<0.3, y<0.6$, в то время как „широкая“ часть InGaAsP/GaP КЯ характеризуется меньшим содержанием атомов In и As - в пределах $x<0.12$ и $y<0.24$.

\section{3. Фотолюминесценция}

Проведены измерения спектров ФЛ полученных гетероструктур с КЯ и эпитаксиальных слоев $\mathrm{GaP} / \mathrm{Si}$. Как ранее нами обсуждалось в работе [13], благодаря эффектам локализации носителей заряда наличие дислокаций не оказывает существенного влияния на люминесцентные свойства гетероструктур, сформированных в эпитаксиальных слоях $\mathrm{GaP} / \mathrm{Si}$. Нормированные спектры низкотемпературной $(5 \mathrm{~K})$ стационарной ФЛ InAs/GaP-гетероструктур с КЯ (измеренные при $P_{\text {ex }}=25 \mathrm{мB} / \mathrm{cm}^{2}$ ) и эпитаксиальных слоев $\mathrm{GaP} / \mathrm{Si}$ (измеренные при $\left.P_{\text {ex }}=25 \mathrm{~B} / \mathrm{cm}^{2}\right)$ представлены на рис. $4, a$. $\mathrm{B}$ спектре ФЛ $\mathrm{GaP}$ слоев присутствует полоса с максимумом на энергии 2.297 эВ и шириной $\sim 4$ мэВ, связанная рекомбинацией экситонов, локализованных на мелких примесях [20,21]. Также присутствует группа полос в диапазоне энергий 2.05-2.27 эВ, связанная с донорно-акцепторной рекомбинацией в $\mathrm{GaP}$ [20]. В спектре InAs/GaP-гетероструктуры доминируют две полосы, отмеченные на рис. 4, $a$ цифрами 1 и 2: с максимумом на энергии 2.14 эВ и шириной 42 мэВ и с максимумом на энергии 2.19эВ и шириной 33 мэВ. Полосы ФЛ InAs/GaP-гетероструктуры лежат в том же диапазоне энергий, что и полосы примесной ФЛ в $\mathrm{GaP}$. Для того чтобы уверенно определить происхождение полос ФЛ в InAs/GaP-структуре, были проведены измерения спектров ФЛ в различных точках образца. Зависимость смещения положения максимумов полос донорноакцепторной рекомбинации в $\mathrm{GaP} / \mathrm{Si}$-слоях и полос ФЛ в спектрах InAs/GaP-гетероструктур от координаты вдоль поверхности образца показана на вставке к рис. 4, $a$. Видно, что структура $\mathrm{GaP} / \mathrm{Si}$ пространственно однородна, в то время как положение полос в InAs/GaP-гетероструктуре монотонно смещается на 20 мэВ. Это указывает на то, что полосы 1 и 2 связаны с рекомбинацией носителей заряда в КЯ. Спектральное смещение полос 1 и 2 обусловлено неоднородностью потока атомов In, 

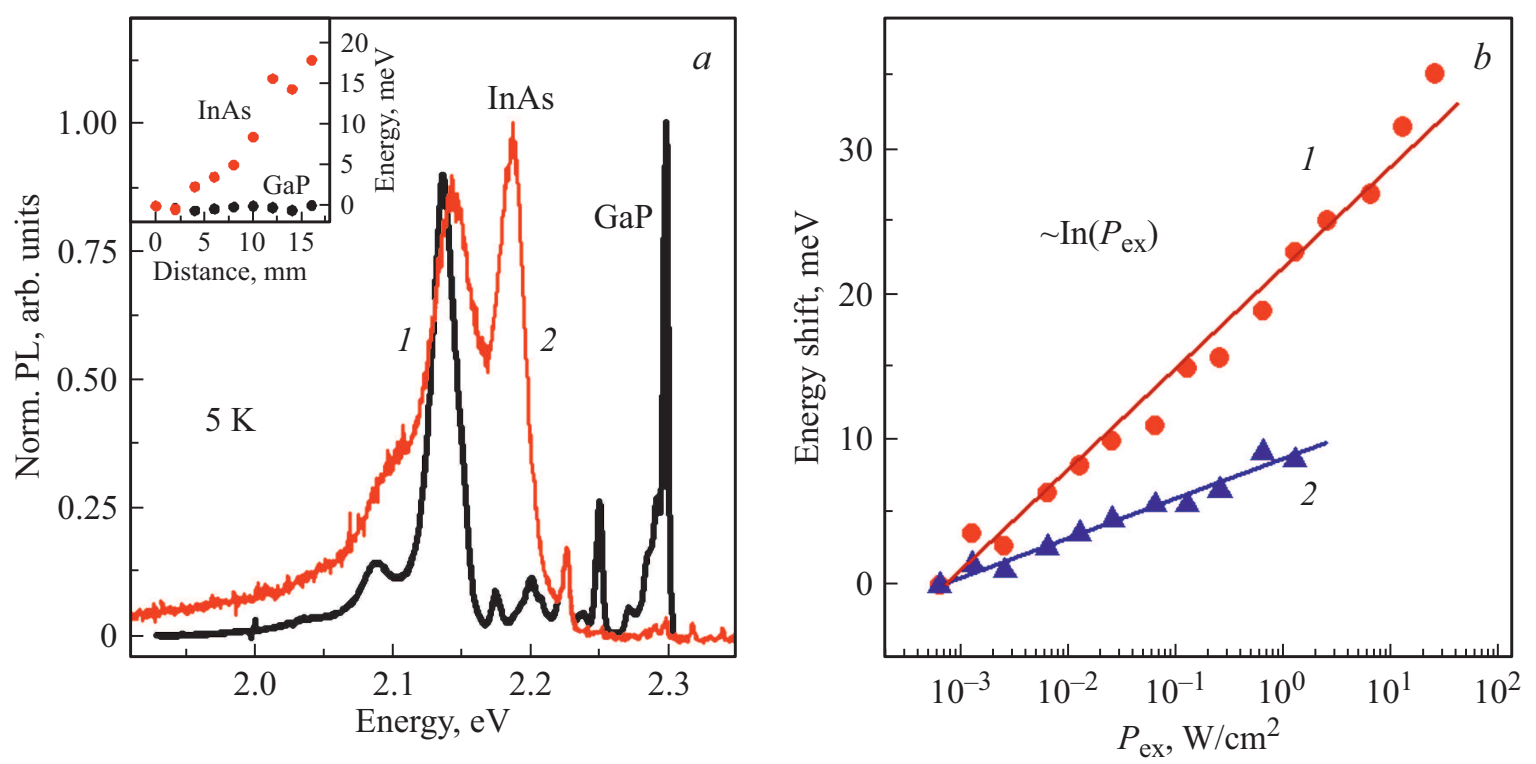

Рис. 4. $a$ - нормированные спектры стационарной низкотемпературной ФЛ структур с $\operatorname{InAs} / \mathrm{GaP}$ КЯ $\left(P_{\mathrm{ex}}=25 \mathrm{MB} / \mathrm{cm}^{2}\right)$ и эпитаксиальных слоев $\mathrm{GaP} / \mathrm{Si}\left(P_{\mathrm{ex}}=25 \mathrm{~B} / \mathrm{cm}^{2}\right)$, измеренные при $5 \mathrm{~K}$. На вставке к рис. 4, $а$ показано спектральное смещение полосы ФЛ InAs/GaP КЯ и примесной ФЛ $\mathrm{GaP}$ в зависимости от смещения вдоль поверхности образца. $b-$ величина спектрального смещения полос ФЛ InAs/GaP КЯ 1 и 2 в зависимости от $P_{\text {ex }}$.

приводящего к градиенту номинальной толщины осажденного InAs, из которого формируется КЯ, по площади гетероструктуры InAs/GaP. Как будет показано далее, полосы ФЛ 1 и 2 связаны с рекомбинацией носителей заряда в участках КЯ, характеризующихся существенно различной толщиной и составом твердого раствора.

Проведены измерения спектров ФЛ InAs/GaP-гетероструктур при различных плотностях мощности возбуждения $\left(P_{\mathrm{ex}}\right)$. С ростом $P_{\text {ex }}$ наблюдается высокоэнергетическое смещение полос ФЛ, связанных с рекомбинацией носителей заряда в КЯ. Зависимость величины смещения от $P_{\text {ex }}$ представлена на рис. $4, b$. Смещение для низкоэнергетической и высокоэнергетической полос ФЛ InAs/GaP-структуры обозначено на рис. $4, b$ как 1 и 2 соответственно. Как видно из рисунка, для всех полос ФЛ эта зависимость хорошо описывается логарифмическим законом $\sim \ln \left(P_{\text {ex }}\right)$. Величина спектрального смещения для полосы $1-6.7 \pm 0.7$ мэВ на декаду, а для $2-$ $2.3 \pm 0.5$ мэВ на декаду. Отметим, что интегральная интенсивность ФЛ сохраняет линейную зависимость от $P_{\text {eх }}$ во всем диапазоне измерений. В соответствии с результатами работы [22] логарифмическое смещение полосы ФЛ КЯ с ростом $P_{\text {ex }}$, при сохранении линейной зависимости интегральной интенсивности ФЛ от $P_{\text {ex }}$, связано с заполнением, „хвостов“ плотности локализованных состояний. Появление этих „хвостов“ вызвано флуктуациями положения уровней размерного квантования в КЯ из-за неоднородности размера и состава КЯ [23].

В конце раздела мы бы хотели подытожить основные полученные экспериментальные результаты.

1. Поверхность $\mathrm{GaP} / \mathrm{Si}$-структур характеризуется развитым рельефом и, как следствие, существенной неод- нородностью в плотности и ориентации монослойных ступеней.

2. B InAs/GaP-гетероструктурах, полученных на $\mathrm{GaP} / \mathrm{Si}$-буферных слоях, происходит формирование псевдоморфно-напряженных КЯ, состоящих из твердого раствора InGaAsP.

3. $\mathrm{In}_{x} \mathrm{Ga}_{1-x} \mathrm{As}_{y} \mathrm{P}_{1-y} / \mathrm{GaP}$ КЯ характеризуется неоднородностью толщины и состава твердого раствора. Наблюдаются участки КЯ с толщиной 1.4 и 4.5 нм. Участки КЯ с меньшей толщиной содержат большую долю атомов In и As: для „узкой“ части КЯ состав лежит в пределах $x<0.3$ и $y<0.6$, а для ,широкой“ $x<0.12$ и $y<0.24$. Латеральные размеры участков КЯ различной толщины составляют не менее $20 \mathrm{Hм}$.

4. В спектрах низкотемпературной ФЛ InAs/GaP-гетероструктур с КЯ, измеренных на участке структуры с латеральными размерами $\sim 300$ мкм, доминируют две полосы с максимумами на энергиях 2.14 и 2.19эВ и шириной 42 и 33 мэВ соответственно.

5. Полосы ФЛ демонстрируют высокоэнергетическое смещение с ростом плотности мощности возбуждения $\sim \ln \left(P_{\mathrm{ex}}\right)$, при этом низкоэнергетическая полоса смещается на $6.7 \pm 0.7$ мэВ на декаду, а высокоэнергетическая — на $2.3 \pm 0.5$ мэВ на декаду.

\section{4. Обсуждение результатов}

Полученные экспериментальные данные демонстрируют наличие двух участков InGaAsP/GaP КЯ с различной толщиной и составом твердого раствора и в то же время наличие двух спектральных полос низкотемпературной стационарной ФЛ. Для того чтобы установить 
соответствие между участками КЯ и полосами ФЛ были проведены расчеты зонных диаграмм и энергии оптического перехода для InGaAsP/GaP КЯ. В ходе расчетов КЯ моделировалась как слой твердого раствора постоянной толщины и состава, помещенный между двумя слоями $\mathrm{GaP}$ и согласованный с ним в плоскости структуры. Учитывалось влияние перемешивания материалов и упругих деформаций на параметры материалов и положение энергетических зон. Влияние перемешивания материалов на параметры твердого раствора учтено в квадратичном приближении [5]:

$$
\begin{aligned}
& P_{\mathrm{ABCD}}=x y P_{\mathrm{AC}}+(1-x) y P_{\mathrm{BC}}+(1-x)(1-y) P_{\mathrm{BD}} \\
& +x(1-y) P_{\mathrm{AD}}+x(1-x) y C_{\mathrm{ABC}}+(1-x) y(1-y) C_{\mathrm{BCD}} \\
& +x(1-x)(1-y) C_{\mathrm{ABD}}+x y(1-y) C_{\mathrm{ACD}},
\end{aligned}
$$

где $P_{\mathrm{ABCD}}$ - параметр четверного твердого раствора $\mathrm{A}_{x} \mathrm{~B}_{1-x} \mathrm{C}_{y} \mathrm{D}_{1-y}, P_{i j}$ - параметр бинарного материала, $C_{i j k}$ - коэффициенты нелинейности для тройного твердого раствора. Деформации были рассчитаны в приближении сплошной среды [19]. Влияние деформаций на положение энергетических зон учтено с помощью деформационных потенциалов. Расчет уровней квантования производился в однозонной модели, путем решения одномерного уравнения Шредингера с эффективной массой, зависящей от координаты. Для дискретизации уравнения Шредингера координатная ось роста была разбита на равные отрезки, в каждом из которых потенциал и эффективная масса предполагались постоянными. На границах этих отрезков использовались граничные условия BenDaniel-Duke [24], т.е. предполагалась непрерывность волновой функции и отношения производной волновой функции к эффективной массе. Уравнение Шредингера решалось методом стрельбы [25]. Параметры бинарных материалов $\mathrm{A}^{\mathrm{III}} \mathrm{B}^{\mathrm{V}}$ (и соответствующие им коэффициенты нелинейности): постоянные решетки, коэффициенты жесткости, ширина запрещенной зоны в $\Gamma, X$ и $L$ точках зоны Брюллиэна, значение разрывов валентных зон и эффективная масса носителей заряда были взяты из работы [5] Деформационные потенциалы были взяты из работ [26,27]. Экситонный эффект в рассмотрение не принимался. Расчеты проведены для КЯ толщиной 1.4 и 4.5 нм и различных составов твердого раствора.

Расчеты показали, что при используемых значениях состава и толщины КЯ основное электронное состояние лежит в $X_{x y}$ подзоне зоны проводимости InGaAsP, a основное дырочное - в зоне тяжелых дырок InGaAsP. Это находится в хорошем согласии с результатами исследований энергетического спектра гетероструктур на основе материалов InAs и GaP, представленных в работе [11]. Расчетные значения энергии оптического перехода для КЯ толщиной 1.4 нм отмечены на рис. 3 возле красных точек, соответствующих составу твердого раствора КЯ. Значения энергий для КЯ толщиной 4.5 нм отмечены возле синих точек на рисунке. Проведено сопоставление расчетной энергии оптического перехода и данных ФЛ с учетом ширины полос ФЛ. Заштрихованными областями на рис. 3 отмечены возможные значения составов твердого раствора, соответствующие КЯ, излучающим на энергии, согласующейся с данными ФЛ. Как видно из рис. 3, низкоэнергетическая полоса ФЛ КЯ, обозначенная 1 на спектрах, соответствует КЯ толщиной 1.4 нм и с составом, лежащим в области $(0.1<x<0.15 ; 0.25<y<0.4)$. Высокоэнергетической полосе ФЛ (2) могут соответствовать две области значений составов: для КЯ толщиной $1.4 \mathrm{HM}-$ $(0.17<x<0.22 ; 0.17<y<0.22)$ и для КЯ толщиной 4.5 нм - $(0<x<0.04 ; 0.18<y<0.23)$. В то же время наблюдаемое в экспериментах по измерению интенсивностной зависимости спектров ФЛ высокоэнергетическое смещение полосы ФЛ 1 , составляющее $6.7 \pm 0.7$ мэВ на декаду, существенно, почти в 3 раза, превосходит величину смещения полосы 2 , составляющее $2.3 \pm 0.5$ мэВ на декаду. Это позволяет выбрать из двух возможных вариантов интерпретации высокоэнергетической полосы ФЛ 2 тот, который соответствует более широкой КЯ, так как для более широкой КЯ смещение уровней размерного квантования при флуктуациях толщины и состава, а значит, и протяженность „хвостов“ плотности локализованных состояний существенно меньше, чем для узкой.

В единичном измерении спектров ФЛ излучение собиралось с области структуры с характерными размерами $\sim 300$ мкм. Кроме того, как уже упоминалось выше, проводились измерения в различных точках гетероструктуры, при этом форма спектра ФЛ не претерпевала существенных изменений. Все это позволяет утверждать, что наблюдаемая на ПЭМ-изображениях картина с разделением КЯ на сегменты различной толщины и состава справедлива в масштабах всей гетероструктуры.

Теперь необходимо обсудить возможные причины возникновения ситуации с разделением КЯ на сегменты различной толщины и состава. Для начала рассмотрим формирование напряженной гетероэпитаксиальной пленки на вицинальной поверхности. Как обсуждается в обзоре [28], осаждение слоев $\mathrm{SiGe}$ на вицинальную поверхность $\mathrm{Si}$ может приводить к самоорганизации

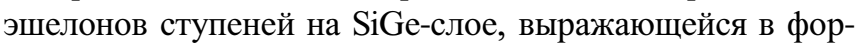
мировании двух типов участков слоя осажденного вещества с различной толщиной. При этом латеральные размеры участков с различной толщиной существенно превышают длину террасы и могут достигать сотен нанометров. Данное явление обусловлено влиянием полей упругих деформаций на скорость движения монослоеных ступеней при росте пленки. Так как в нашем случае мы имеем дело с террасированной поверхностью $\mathrm{GaP}$, то резонно предположить возможность протекания схожих процессов при осаждении InAs на GaP с той разницей, что сегменты КЯ различной толщины имеют форму, задаваемую рельефом поверхности GaP. В ходе заращивания сформировавшегося слоя InAs материалом матрицы в поверхностной диффузии принимают 
участи как адатомы $\mathrm{Ga}$ и $\mathrm{P}$, так и атомы In и As. Появление атомов In и As на ростовой поверхности может быть обусловлено как процессами сегрегации атомов III группы [29-33], так и реакцией замещения P/As [34-36]. В работе [37] обсуждается, что при росте твердого раствора на террасированной поверхности возможен латеральный массоперенос атомов, приводящий к формированию двух типов областей структуры с различным составом. Такое расслоение слоя растущего твердого раствора обусловлено влиянием локальных деформаций на эффективность встраивания атомов различных видов [38]. Таким образом, у нас есть основания предполагать, что наблюдаемое разделение КЯ на сегменты двух типов (различной толщины и состава) вызвано процессами перестройки поверхности в ходе гетероэпитаксии InAs на террасированной поверхности GaP.

\section{5. Заключение}

Проведены исследования процессов формирования и строения InAs/GaP-гетероструктур, выращенных на $\mathrm{GaP} / \mathrm{Si}$ эпитаксиальных слоях с развитым рельефом поверхности. Показано, что при осаждении InAs в количестве $2 \mathrm{MC}$ на такую поверхность $\mathrm{GaP}$ возможно формирование псевдоморфно-напряженной КЯ, состоящей из четверного твердого раствора $\operatorname{In}_{x} \mathrm{Ga}_{1-x} \mathrm{As}_{y} \mathrm{P}_{1-y}$, при этом образования самоорганизованных КТ не происходит. Полученная КЯ характеризуется наличием двух типов участков с различной толщиной и составом твердого раствора, которым соответствуют различные полосы низкотемпературной стационарной ФЛ. Сопоставление экспериментальных данных ПЭМ, ФЛ и результатов расчетов энергии оптического перехода КЯ показало, что увеличение толщины КЯ с 1.4 до 4.5 нм сопровождается снижением содержания атомов In и As c $0.1<x<0.15$ и $0.25<y<0.4$ до $0<x<0.04$ и $0.18<y<0.23$. Изменение параметров КЯ объяснено в рамках предположения о перестройке поверхности под действием упругих деформаций при гетероэпитаксии InAs на террасированной поверхности GaP.

\section{Финансирование работы}

Исследование выполнено при финансовой поддержке РФФИ и Министерства науки и инновационной политики Новосибирской области в рамках научного проекта № 19-42-543009. ВРЭМ исследования проводились на оборудовании ЦКП „Наноструктуры“ при поддержке РНФ (проект 19-72-30023).

\section{Конфликт интересов}

Авторы заявляют, что у них нет конфликта интересов.

\section{Список литературы}

[1] D. Liangand, J.E. Bowers. Nature Photonics, 4, 511 (2010).

[2] M. Asghari, A.V. Krishnamoorth. Nature Photonics, 5, 268 (2011).

[3] A. Rickman. Nature Photonics, 8, 579 (2014).

[4] Ch. Sun, M.T. Wade, Y. Lee, J.S. Orcutt, L. Alloatti, M.S. Georgas, A.S. Waterman, J.M. Shainline, R.R. Avizienis, S. Lin, B.R. Moss, R. Kumar, F. Pavanello, A.H. Atabaki, H.M. Cook, A.J. Ou, J.C. Leu, Y.-H. Chen, K. Asanović, R.J. Ram, M.A. Popović, V.M. Stojanović. Nature, 528 (7583), 534 (2015).

[5] I. Vurgaftman, J.R. Meyer, L.R. Ram-Mohan. J. Appl. Phys., 89, 5815 (2001).

[6] M. Heidemann, S. Hofling, M. Kamp. Appl. Phys. Lett., 104, 011113 (2014).

[7] G. Stracke, E. M. Sala, S. Selve, T. Niermann, A. Schliwa, A. Strittmatter, D. Bimberg. Appl. Phys. Lett., 104, 123107 (2014).

[8] S. Dadgostar, J. Schmidtbauer, T. Boeck, A.Torres, O. Martinez, J. Jimenez, J.W. Tomm, A. Mogilatenko, W.T. Masselink, F. Hatami. Appl. Phys. Lett., 108, 102103 (2016).

[9] D.S. Abramkin, M.A. Putyato, S.A. Budennyy, A.K. Gutakovskii, B.R. Semyagin, V.V. Preobrazhenskii, O.F. Kolomys, V.V. Strelchuk, T.S. Shamirzaev. J. Appl. Phys., 112, 083713 (2012).

[10] C. Robert, K. Pereira Da Silva, M.O. Nestoklon, M.I. Alonso, P. Turban, J.-M. Jancu, J. Even, H. Carrére, A. Balocchi, P.M. Koenraad, X. Marie, O. Durand, A.R. Goñi, C. Cornet. Phys. Rev. B, 94, 075445 (2016).

[11] C. Robert, C. Cornet, P. Turban, T. Nguyen Thanh, M.O. Nestoklon, J. Even, J.M. Jancu, M. Perrin, H. Folliot, Rohel, S. Tricot, A. Balocchi, D. Lagarde, X. Marie, N. Bertru, O. Durand, A. Corre. Phys. Rev. B, 86, 205316 (2012).

[12] Yu. Song, M.L. Lee. Appl. Phys. Lett., 103, 141906 (2013).

[13] Д.С. Абрамкин, М.О. Петрушков, М.А. Путято, Б.Р. Семягин, Е.А. Емельянов, В.В. Преображенский, А.К. Гутаковский, Т.С. Шамирзаев. ФТП, 53 (9), 1167 (2019).

[14] T. Sakamoto, G. Hashiguchi. Jpn. J. Appl. Phys., 25 (1A), L78 (1986).

[15] D.J. Chadi. Phys. Rev. Lett., 59 (15), 1691 (1987).

[16] Y. Okada, Y. Tokumaru. J. Appl. Phys., 56, 314 (1984).

[17] V. Narayanan, S. Mahajan, N. Sukidi, K.J. Bachmann, V. Woods, N. Dietz. Phil. Mag. A, 80 (3), 555 (2000).

[18] R. Leon, C. Lobo, T.P. Chin, S. Fafard, S. Ruvimov, Z. Liliental-Weber, M.A. Stevens Kalceff. Appl. Phys. Lett., 72, 1356 (1998).

[19] C.G. Vande Walle. Phys. Rev. B, 39, 1871 (1989).

[20] A.T. Vink, A.J. Bosman, J.A. van der Does de Bye, R.C. Peters. Solid State Commun., 7, 1475 (1969).

[21] Е.Ф. Гросс, Д.С. Недзвецкий. ДАН СССР, 152, 309 (1963).

[22] D.S. Abramkin, A.K. Gutakovskii, T.S. Shamirzaev. J. Appl. Phys., 123, 115701 (2018).

[23] A. Klochikhin, A. Reznitsky, S. Permogorov, T. Breitkopf, M. Grun, M. Hetterich, C. Klingshirn, V. Lyssenko, W. Langbein, J.M. Hvam. Phys. Rev. B, 59, 12947 (1999).

[24] D.J. BenDaniel, C.B. Duke. Phys. Rev., 152, 683 (1966).

[25] Н.Н. Калиткин. Численные методы (М., Наука, 1978).

[26] S.H. Wei, A. Zunger. Appl. Phys. Lett., 72, 2011 (1998).

[27] M.C. Munoz, G. Armelles. Phys. Rev. B, 48, 2839 (1993).

[28] Ch. Teichert. Phys. Rep., 365, 335 (2002). 
[29] K. Muraki, S. Fukatsu, Y. Shiraki. Appl. Phys. Lett., 61, 557 (1992).

[30] S. Fukatsu, K. Fujita, H. Yaguchi, Y. Shiraki, R. Ito. Appl. Phys. Lett., 59, 2103 (1991).

[31] O. Dehaese, X. Wallart, F. Mollot. Appl. Phys. Lett., 66, 52 (1995).

[32] D.J. Godbey, M. G. Ancona. J. Vac. Sci. Technol. A, 15, 976 (1997).

[33] V. Haxha, I. Drouzas, J.M. Ulloa, M. Bozkurt, P.M. Koenraad, D.J. Mowbray, H.Y. Liu, M.J. Steer, M. Hopkinson, M.A. Migliorato. Phys. Rev. B, 80, 165334 (2009).

[34] E.E. Mura, A. Gocalinska, G. Juska, S.T. Moroni, A. Pescaglini, E. Pelucchi. Appl. Phys. Lett., 110, 113101 (2017).

[35] M.J.S.P. Brasil, R.E. Nahory, M.C. Tamargo, S.A. Schwarz. Appl. Phys. Lett., 63, 2688 (1993).

[36] M. Taskinen, M. Sopanen, H. Lipsanen, J. Tulkki, T. Tuomi, J. Ahopelto. Surf. Sci., 376, 60 (1997).

[37] P. Venezuela, J. Terso, J.A. Floro, E. Chason, D.M. Follstaedt, F. Liu, M.G. Lagally. Nature, 397, 678 (1999).

[38] J. Tersoff. Phys. Rev. Lett., 77, 2017 (1996).

Редактор А.Н. Смирнов

\section{Formation of InAs/GaP heterostructures with quantum wells on silicon substrates by molecular beam epitaxy}

D.S. Abramkin ${ }^{1,2}$, M.O. Petrushkov ${ }^{1}$, E.A. Emelyanov ${ }^{1}$, A.V. Nenashev ${ }^{1,2}$, M.Yu. Yesin ${ }^{1}$, A.V. Vasev ${ }^{1}$, M.A. Putyato ${ }^{1}$, D.B. Bogomolov ${ }^{1}$, A.K. Gutakovskiy ${ }^{1,2}$, V.V. Preobrazhenskiy ${ }^{1}$

${ }^{1}$ Rzhanov Institute of Semiconductor Physics, Siberian Branch of Russian Academy of Sciences, 630090 Novosibirsk, Russia

${ }^{2}$ Novosibirsk State University, 630090 Novosibirsk, Russia

Abstract Possibility of formation of pseudomorphous quantum well consisting of $\operatorname{In}_{x} \mathrm{Ga}_{1-x} \mathrm{As}_{y} \mathrm{P}_{1-y}$ quaternary alloy during InAs deposition on $\mathrm{GaP} / \mathrm{Si}$ epitaxial film surface with developed relief is demonstrated. Investigations of quantum well were performed by transmission electron microscopy and spectroscopy of $\mathrm{cw}$ photoluminescence. The appearance of quantum well segments of 2 types with different width and composition $\operatorname{In}_{x} \mathrm{Ga}_{1-x} \mathrm{As}_{y} \mathrm{P}_{1-y}$ is demonstrated. Width increasing is accompanied by decreasing of In and As atoms fraction. Lateral sizes of quantum well segments are not lower than $20 \mathrm{~nm}$. Different photoluminescence bands are corresponds to quantum well segments. Observed phenomenon are explained in the framework of suggestion about straininduced surface reorganization during InAs heteroepitaxy on terraced GaP surface. 